\title{
OUTER MEASURES AND WEAK TYPE $(1,1)$ ESTIMATES OF HARDY-LITTLEWOOD MAXIMAL OPERATORS
}

\author{
YUTAKA TERASAWA
}

Received 14 June 2004; Revised 10 July 2004; Accepted 14 October 2004

We will introduce the $k$ times modified centered and uncentered Hardy-Littlewood maximal operators on nonhomogeneous spaces for $k>0$. We will prove that the $k$ times modified centered Hardy-Littlewood maximal operator is weak type $(1,1)$ bounded with constant 1 when $k \geq 2$ if the Radon measure of the space has "continuity" in some sense. In the proof, we will use the outer measure associated with the Radon measure. We will also prove other results of Hardy-Littlewood maximal operators on homogeneous spaces and on the real line by using outer measures.

Copyright (c) 2006 Yutaka Terasawa. This is an open access article distributed under the Creative Commons Attribution License, which permits unrestricted use, distribution, and reproduction in any medium, provided the original work is properly cited.

\section{Introduction}

Hardy-Littlewood maximal operators were first introduced by Hardy and Littlewood ([6]) in one dimensional case for the purpose of the application to Complex Analysis. Then Wiener ([14]) introduced this operator in higher dimensional Eucledian spaces for the purpose of the application to Ergodic Theory. Later, Coifman and Weiss ([4]) defined Hardy-Littlewood maximal operators on quasi-metric measure spaces satisfying doubling conditions (which we call homogeneous spaces). More recently, Nazarov et al. ([9]) defined modified Hardy-Littlewood maximal operators on quasi-metric measure spaces possesing a Radon measure that does not satisfy a doubling condition (which we call nonhomogeneous spaces), which are used in harmonic analysis on nonhomogeneous spaces. In this paper, we will treat weak type $(1,1)$ inequalities satisfied by several types of Hardy-Littlewood maximal operators. As is well known, weak type $(1,1)$ inequalities satisfied by Hardy-Littlewood maximal operators are keys to prove their strong type $(p, p)$ boundedness via Marcinkiewicz's interpolation theorem. To prove their weak type $(1,1)$ inequalities, the unification of our approach is the use of outer measures. The advantage of the use of outer measures over usual measures is that they could measure any subsets of a total space, even when they are nonmeasurable. 
Let $(X, \mu)$ be a metric space possesing a nondegenerate Radon measure such that $\mu(B(x, r))$ is continuous with respect to the variable $r>0$ when the variable $x \in X$ is fixed, where $B(x, r)$ denotes a ball centered at $x$ and of radius $r$. We will define the $k$ times modified centered Hardy-Littlewood maximal operator as follows:

$$
M_{k} f(x)=\sup _{r>0} \frac{1}{\mu(B(x, k r))} \int_{B(x, r)}|f(y)| d \mu(y) .
$$

We will prove that the $k$ times modified centered Hardy-Littlewood maximal operator $M_{k}$ is weak- $(1,1)$ bounded when $k$ is larger than or equal to 2 , and that their weak- $(1,1)$ constant (which is the infimum (consequently the minimum) of the constant appearing in the weak type $(1,1)$ inequality) is less than or equal to 1 . We will state the main idea of the proof of this fact. Let $R>0$ be fixed. Let $k>0$. We consider the $k$ times modified centered Hardy-Littlewood maximal operator with bounded radius:

$$
M_{k, R} f(x)=\sup _{r \leq R} \frac{1}{\mu(B(x, k r))} \int_{B(x, r)}|f(y)| d \mu(y) .
$$

We set $A_{\lambda}:=\left\{x \mid M_{k, R} f(x)>\lambda\right\}$. The set $A_{\lambda}$ is easily seen to be an open set. From the continuity of the measure, we can assume that $k>2$. Let $J \subset A_{\lambda}$ be an arbitrary compact set. For each $x \in J$, we choose $r_{x}$ such that

$$
\frac{1}{\mu\left(B\left(x, k r_{x}\right)\right)} \int_{B\left(x, r_{x}\right)}|f(y)| d \mu(y)>\lambda
$$

Set

$$
J_{n}:=\left\{x \in J \mid r_{x}>\frac{1}{n}\right\}
$$

The set $J_{n}$ is not necessarily measurable. So we use the outer measure associated with $\mu$ to estimate the "size" of the set $J_{n}$. Take $0<\theta<1$ such that $1<(k-1) \theta$. Set

$$
R_{1}:=\sup _{x \in J_{n}} r_{x}
$$

Then there exists $x_{1} \in J_{n}$ such that $\theta R_{1}<r_{x_{1}}$, and it holds that

$$
\frac{1}{\lambda} \int_{B\left(x_{1}, r_{x_{1}}\right)}|f(y)| d \mu(y)>\mu\left(B\left(x_{1}, k r_{x_{1}}\right)\right)
$$

If $B\left(x_{1}, k r_{x_{1}}\right) \supset J_{n}$, then we have $\mu^{*}\left(J_{n}\right) \leq 1 / \lambda\|f\|_{1}$. Here, $\mu^{*}$ is the outer measure associated with $\mu$, that is,

$$
\mu^{*}(B)=\inf _{B \subseteq C, C: \text { measurable }} \mu(C) .
$$


If $B\left(x_{1}, k r_{x_{1}}\right) \not \supset J_{n}$, we set

$$
R_{2}:=\sup _{x \in J_{n} \backslash B\left(x_{1}, k r_{x_{1}}\right)} r_{x}
$$

We proceed in the same way. This process ends in finite times, because of the compactness of $J$ and the lower uniform boundness of $r_{x}$. Thus we obtain the proof.

Furthermore, we will treat the weighted weak- $(1,1)$ inequality of the centered HardyLittlewood maximal operator on a metric space possesing a doubling Radon measure. We will get some upper bound of the weak- $(1,1)$ constant of the weighted weak- $(1,1)$ inequality of the centered Hardy-Littlewood maximal operator. We should remark that the method of this proof resembles to that of the above mentioned result on nonhomogeneous spaces.

The following is the constitution of our paper.

In Section 2, we will prove weak- $(1,1)$ boundedness of the $k$ times modified centered Hardy-Littlewood maximal operators on nonhomogeneous spaces with measures which have "continuities" in some sense when $k$ is larger than 2. (We will state what is meant by the word "continuities" later.) After our result, Sawano ([10]) proved a result of the same type in the setting of a separable metric space without this continuity assumption.

In Section 3, we will prove weak- $(1,1)$ boundedness of centered Hardy-Littlewood maximal operators under $A_{1}$-weights (the definition of which we will state later) with better constants than are previously known (as far as we know). The weak- $(1,1)$ norm of the centered Hardy-Littlewood maximal operator on the real line is recently determined by Melas ([7]). Our result may be regarded as some upper bound estimates of the weak- $(1,1)$ norms of the centered Hardy-Littlewood maximal operator on homogeneous spaces under general $A_{1}$-weights.

In Section 4, we will prove weak- $(1,1)$ norms of one-sided Hardy-Littlewood maximal operators on the real line with absolutely continuous measure are less than or equal to 1 . Bernal ([1]) proved more general results under only assumptions that the measures on the real line are Borel. We will give a different proof of special cases of A. Bernal's result. In fact, this kind of proof of the result is already known (cf. $[8,11,14]$ ). However, we include this proof here since this kind of proof of the result may be regarded as the easiest example of our method.

\section{Modified Hardy-Littlewood maximal operators on nonhomogeneous spaces}

To fix the terminology, we will include here the definition of the Hardy-Littlewood maximal operators on a metric measure space. We will consider Hardy-Littlewood maximal operators on a metric measure space $X$ possesing a nondegenerate Radon measure $\mu$ which we will denote as $(X, \mu)$. Here a Radon measure means a measure which is defined on a $\sigma$-algebra on $X$ including all Borel sets and which is inner regular on open sets and outer regular on Borel sets. A nondegenerate Radon measure is a Radon measure such that the measure of balls which have positive radius are positive. We will also assume here that the measures of balls which have finite radius are finite.

There are two types of the Hardy-Littlewood maximal operators, namely the centered one and the uncentered one. We will recall the definition of these here. 
Definition 2.1. Let $(X, \mu)$ be a metric space possesing a nondegenerate Radon measure. Let $f$ be a locally integrable function on $(X, \mu)$. The centered Hardy-Littlewood maximal function Mf of $f$ is defined as follows:

$$
\operatorname{Mf}(x)=\sup _{r>0} \frac{1}{\mu(B(x, r))} \int_{B(x, r)}|f(y)| d \mu(y) .
$$

We call the operator $M$ associating $f$ to Mf the centered Hardy-Littlewood maximal operator. Next, we define the uncentered Hardy-Littlewood maximal operator. The uncentered Hardy-Littlewood maximal function $M_{u c} f$ of the locally integrable function $f$ is defined as

$$
M_{u c} f(x)=\sup _{x \in B(y, r)} \frac{1}{\mu(B(y, r))} \int_{B(y, r)}|f(z)| d \mu(z) .
$$

We call the operator $M_{u c}$ associating $f$ to $M_{u c} f$ the uncentered Hardy-Littlewood maximal operator.

Let us assume that $(X, \mu)$ satisfies a doubling condition, and let $C$ be their doubling constant. Then, for any locally integrable function on $(X, \mu)$, the inequalities $\mathrm{Mf} \leq M_{u c} f$ and $M_{u c} f \leq C^{2}$. Mf holds pointwise. The centered Hardy-Littlewood maximal operator $M$ and the uncentered one $M_{u c}$ are both weak type $(1,1)$ and strong type $(p, p)$ $(1<p \leq+\infty)$. We can prove that the operators $M$ and $M_{u c}$ are both strong type $(p, p)$ $(1<p<+\infty)$ from the fact that they are weak type $(1,1)$ and strong type $(+\infty,+\infty)$ by using Marcinkiewicz's interpolation theorem. It is trivial that $M$ and $M_{u c}$ are both strong type $(+\infty,+\infty)$, so the problem is to prove that they are weak type $(1,1)$. Since $\operatorname{Mf}(x) \leq M_{u c} f(x)$, it suffices to prove that $M_{u c}$ is weak type $(1,1)$. We can prove that $M_{u c}$ is weak type $(1,1)$ by using the following (finite type) Vitali's covering lemma.

Theorem 2.2. Let $X$ be a metric space and let a finite collection of balls $\left\{B\left(x_{k}, r_{k}\right)\right\}_{k=1}^{k=n}$ be given. Then we can find a subcollection of balls $\left\{B\left(x_{k_{i}}, r_{k_{i}}\right)\right\}_{i=1}^{i=j}$ which are mutually disjoint such that $\bigcup_{k=1}^{k=n} B\left(x_{k}, r_{k}\right) \subset \bigcup_{i=1}^{i=j} B\left(x_{k_{i}}, 3 r_{k_{i}}\right)$ holds.

F. Nazarov, S. Treil and A. Volberg introduced a type of modified Hardy-Littlewood maximal operators on nonhomogeneous spaces. We will introduce the $k$ times modified centered Hardy-Littlewood maximal operators and $k$ times modified uncentered HardyLittlewood maximal operators.

Definition 2.3. Let $f$ be a locally integrable function on a metric measure space $(X, \mu)$. Then the $k$ times modified centered Hardy-Littlewood maximal function $M_{k} f$ of $f$ is defined as follows:

$$
M_{k} f(x)=\sup _{r>0} \frac{1}{\mu(B(x, k r))} \int_{B(x, r)}|f(y)| d \mu(y) .
$$

We call the operator $M_{k}$ the $k$ times modified centered Hardy-Littlewood maximal operator. The $k$ times modified uncentered Hardy-Littlewood maximal function $M_{k} f$ of $f$ is 
defined as follows:

$$
M_{k, u c} f(x)=\sup _{x \in B(y, r)} \frac{1}{\mu(B(y, k r))} \int_{B(y, r)}|f(z)| d \mu(z) .
$$

We call the operator $M_{k, u c}$ the $k$ times modified uncentered Hardy-Littlewood maximal operator.

As is easily seen, the pointwise inequalities $M_{k} f \leq M_{k^{\prime}} f\left(k^{\prime} \leq k\right)$ and $M_{k, u c} f \leq M_{k^{\prime}, u c} f$ $\left(k^{\prime} \leq k\right)$ holds for any locally integrable function $f$ on $(X, \mu) . M_{k, u c} f(x)$ is lower semicontinuous for any locally integrable function $f$. We can easily prove that $M_{3, u c}$ is weak- $(1,1)$ bounded by using Vitali's covering lemma. Note that modified Hardy-Littlewood maximal operators introduced by F. Nazarov, S. Treil and A. Volberg are $M_{3} f$ in our notations and that they proved $M_{3}$ is weak- $(1,1)$ bounded.

Let $X$ be a metric space possesing a nondegenerate Radon measure $\mu$ such that the measure is "continuous" in the sense that $\mu(B(x, r))$ is continuous with the variable $r>0$ when $x \in X$ is fixed. Then we can show that $M_{k} f(x)=\sup _{r>0} 1 /(\mu(B(x, k r))) \int_{B(x, r)}|f(y)|$ $d \mu(y)$ is weak- $(1,1)$ bounded with constant 1 when $k$ is larger than 2 . In the course of the proof, we will meet subsets of $X$ which are not necessarily measurable. So we cannot use measures to estimate "sizes" of these sets. So, we use instead an outer measure to estimate "sizes" of these sets.

Theorem 2.4. Let $X$ be a metric space possesing a nondegenerate Radon measure $\mu$ such that $\mu(B(x, r))$ is continuous with the variable $r>0$ when $x \in X$ is fixed. Then $M_{k} f(x)=$ $\sup _{r>0} 1 /(\mu(B(x, k r))) \int_{B(x, r)}|f(y)| d \mu(y)$ is weak- $(1,1)$ bounded with constant 1 when $k$ is larger than or equal to two.

Namely,

$$
\mu\left(\left\{x \mid M_{k} f(x)>\lambda\right\}\right) \leq \frac{1}{\lambda} \int_{X}|f(y)| d y
$$

for any $f \in L^{1}(X, \mu)$ when $k \geq 2$.

Proof. Let $R>0$ be fixed. Let $k>0$. We consider the centered Hardy-Littlewood maximal operator with bounded radius:

$$
M_{k, R} f(x):=\sup _{r \leq R} \frac{1}{\mu(B(x, k r))} \int_{B(x, r)}|f(y)| d \mu(y) .
$$

We set $A_{\lambda}:=\left\{x \mid M_{k, R} f(x)>\lambda\right\}$. We will show that $A_{\lambda}$ is an open set. Let us assume that $x_{0} \in\left\{x \mid M_{k, R} f(x)>\lambda\right\}$. Then there exists $r \leq R$ such that

$$
\frac{1}{\mu\left(B\left(x_{0}, k r\right)\right)} \int_{B\left(x_{0}, r\right)}|f(y)| d \mu(y)>\lambda
$$

By the absolute continuity of the integral, there exists a compact set $K \subset B\left(x_{0}, r\right)$ such that

$$
\frac{1}{\mu\left(B\left(x_{0}, k r\right)\right)} \int_{K}|f(y)| d \mu(y)>\lambda .
$$


If we take $\delta$ sufficiently small, then for any $y$ satisfying $\left|y-x_{0}\right|<\delta$, it holds that $K \subset$ $B(y, r)$ and that

$$
\lambda<\frac{1}{\mu(B(y, k r))} \int_{K}|f(y)| d \mu(y) \leq \frac{1}{\mu(B(y, k r))} \int_{B(y, r)}|f(y)| d \mu(y) .
$$

Therefore $\left\{x \in X \mid M_{k, R} f(x)>\lambda\right\}$ is an open set. Entirely similarly, we can show that $\left\{x \mid M_{k} f(x)>\lambda\right\}$ is an open set.

Since $\mu(B(x, r))$ is continuous with the variable $r>0$ when $x \in X$ is fixed, we have $\left\{x \mid M_{2} f(x)>\lambda\right\}=\bigcup_{k>2}\left\{x \mid M_{k} f(x)>\lambda\right\}$. So we have only to prove the theorem in the case $k>2$. Let $J \subset A_{\lambda}$ be an arbitrary compact set. For each $x \in J$, we choose $r_{x}$ such that

$$
\frac{1}{\mu\left(B\left(x, k r_{x}\right)\right)} \int_{B\left(x, r_{x}\right)}|f(y)| d \mu(y)>\lambda
$$

Set

$$
J_{n}:=\left\{x \in J \mid r_{x}>\frac{1}{n}\right\}
$$

Take $0<\theta<1$ such that $1<(k-1) \theta$. Set

$$
R_{1}:=\sup _{x \in J_{n}} r_{x}
$$

Then there exists $x_{1} \in J_{n}$ such that $\theta R_{1}<r_{x_{1}}$, and it holds that

$$
\frac{1}{\lambda} \int_{B\left(x_{1}, r_{x_{1}}\right)}|f(y)| d \mu(y)>\mu\left(B\left(x_{1}, k r_{x_{1}}\right)\right) .
$$

If $B\left(x_{1}, k r_{x_{1}}\right) \supset J_{n}$, then we have $\mu^{*}\left(J_{n}\right) \leq 1 / \lambda\|f\|_{1}$. Here, $\mu^{*}$ is the outer measure associated with $\mu$, that is,

$$
\mu^{*}(B)=\inf _{B \subseteq C, C: \text { measurable }} \mu(C) .
$$

If $B\left(x_{1}, k r_{x_{1}}\right) \not \supset J_{n}$, we set

$$
R_{2}:=\sup _{x \in J_{n} \backslash B\left(x_{1}, k r_{x_{1}}\right)} r_{x}
$$

Then there exists $x_{2} \in J_{n} \backslash B\left(x_{1}, k r_{x_{1}}\right)$ such that $\theta R_{2}<r_{x_{2}}$, and it holds that

$$
\frac{1}{\lambda} \int_{B\left(x_{2}, r_{x_{2}}\right)}|f(y)| d y>\mu\left(B\left(x_{2}, k r_{x_{2}}\right)\right) .
$$

We should remark that

$$
r_{x_{1}}+r_{x_{2}}<k r_{x_{1}}
$$


In fact

$$
(k-1) r_{x_{1}}-r_{x_{2}}=\frac{1}{\theta}\left((k-1) \theta r_{x_{1}}-\theta r_{x_{2}}\right) \geq \frac{1}{\theta}\left((k-1) \theta r_{x_{1}}-r_{x_{1}}\right)>0 .
$$

Using this, we can show that $B\left(x_{1}, r_{x_{1}}\right) \cap B\left(x_{2}, r_{x_{2}}\right)=\varnothing$. If $B\left(x_{1}, r_{x_{1}}\right) \cap B\left(x_{2}, r_{x_{2}}\right) \neq \varnothing$, $d\left(x_{1}, x_{2}\right) \leq r_{x_{1}}+r_{x_{2}}<k r_{x_{1}}$. This will contradict the fact that $x_{2} \in J_{n} \backslash B\left(x_{1}, k r_{x_{1}}\right)$. Therefore $B\left(x_{1}, r_{x_{1}}\right) \cap B\left(x_{2}, r_{x_{2}}\right)=\varnothing$. If $J_{n} \subset B\left(x_{1}, k r_{x_{1}}\right) \cup B\left(x_{2}, k r_{x_{2}}\right)$, we have $\mu^{*}\left(J_{n}\right) \leq 1 / \lambda\|f\|_{1}$. If $J_{n} \not \subset B\left(x_{1}, k r_{x_{1}}\right) \cup B\left(x_{2}, k r_{x_{2}}\right)$, we set

$$
R_{3}:=\sup _{x \in J_{n} \backslash\left(B\left(x_{1}, k r_{x_{1}}\right) \cup B\left(x_{2}, k r_{x_{2}}\right)\right)} r_{x} .
$$

Then there exists $x_{3} \in J_{n} \backslash\left(B\left(x_{1}, k r_{x_{1}}\right) \cup B\left(x_{2}, k r_{x_{2}}\right)\right)$ such that $\theta R_{3}<r_{x_{3}}$, and it holds that

$$
\frac{1}{\lambda} \int_{B\left(x_{3}, r_{x_{3}}\right)}|f(y)| d y>\mu\left(B\left(x_{3}, k r_{x_{3}}\right)\right) .
$$

We can show that $B\left(x_{1}, r_{x_{1}}\right) \cap B\left(x_{3}, r_{x_{3}}\right)=\varnothing$ and $B\left(x_{2}, r_{x_{2}}\right) \cap B\left(x_{3}, r_{x_{3}}\right)=\varnothing$ in the same manner as before. If $J_{n} \subset B\left(x_{1}, k r_{x_{1}}\right) \cup B\left(x_{2}, k r_{x_{2}}\right) \cup B\left(x_{3}, k r_{x_{3}}\right)$, we have $\mu^{*}\left(J_{n}\right) \leq 1 / \lambda\|f\|_{1}$. We repeat this process. Then, finally, we have

$$
J_{n} \subset B\left(x_{1}, k r_{x_{1}}\right) \cup B\left(x_{2}, k r_{x_{2}}\right) \cup \cdots \cup B\left(x_{l}, k r_{x_{l}}\right) .
$$

For, if not, we can take an infinite sequence $\left\{x_{m}\right\}$ in $J$ which satisfies $d\left(x_{m_{1}}, x_{m_{2}}\right) \geq 1 / n$ $\left(m_{1} \neq m_{2}\right)$. This, however, contradicts the compactness of $J$. Thus we have $\mu^{*}\left(J_{n}\right) \leq$ $1 / \lambda\|f\|_{1}$. Letting $n \rightarrow+\infty$, we have $\mu(J) \leq 1 / \lambda\|f\|_{1}$. Here we use the fact that

$$
\lim _{n \rightarrow+\infty} \mu^{*}\left(J_{n}\right)=\mu^{*}(J)
$$

(For the proof of (2.22), see Lemma 2.8 at the end of this section.) Since $J$ is an arbitrary compact set contained in $A_{\lambda}$, we have $\mu\left(A_{\lambda}\right) \leq 1 / \lambda\|f\|_{1}$ by the inner regularity of $\mu$. Since the right-hand side is independent of $R>0$, we have

$$
\mu\left(\left\{x \in X \mid M_{k} f(x)>\lambda\right\}\right) \leq \frac{1}{\lambda} \int_{X}|f(y)| d y .
$$

Remark 2.5. After our result, Sawano ([10]) proved the following theorem.

Theorem 2.6. Let $X$ be a separable metric space with nondegenerate Radon measure. Then the two times modified centered modified Hardy-Littlewood maximal operators $M_{2}$ as is defined above is weak- $(1,1)$ bounded with constant 1 . Namely, the following inequality holds.

$$
\mu\left(\left\{x \in X \mid M_{2} f(x)>\lambda\right\}\right) \leq \frac{1}{\lambda} \int_{X}|f(y)| d y
$$

for any $f \in L^{1}(X, \mu)$. Furthermore, this result is sharp in the following sense. There exists a separable metric space with nondegenerate Radon measure such that $M_{k}$ is not weak- $(1,1)$ bounded for all positive $k$ smaller than 2. 
He proved this theorem by some variant of Vitali's covering lemma and Lindelöf's covering lemma. He did not use outer measure which we used to prove this theorem. He showed the sharpness of the result by using Kolmogorov's extension law in measure theory. Furthermore, using this theorem, he proved some type of vector-valued inequalities of singular integral operators and Fefferman-Stein's vector-valued version of Hardy-Littlewood maximal inequality on nonhomogeneous spaces. For details, the reader should refer to [10].

Remark 2.7. For completeness, we will include the proof of the following lemma. The following lemma is from [5].

LEMmA 2.8. Let $Y$ be a measure space with a measure $\mu$. Let $\mu^{*}$ is the outer measure associated to the measure $\mu$, that is,

$$
\mu^{*}(B)=\inf _{B \subseteq C, C: \text { measurable }} \mu(C)
$$

for any subset $B$ in $X$. Let $J$ be a measurable set in $Y$. Let $J_{k}(k \geq 1)$ be subsets (which are not necessarily measurable) in $J$ which are increasing in $k$, that is, $J_{k} \subset J_{k+1}$ for any $k \geq 1$.

Proof. From the definition of $\mu^{*}$, for any $A \subset X$, there exists a $\mu$-measurable set $C$ such that $A \subset C$ and $\mu^{*}(A)=\mu(C)$. Therefore for each $J_{k}$, there exists a $\mu$-measurable set $C_{k}$ such that $J_{k} \subset C_{k}$ and $\mu^{*}\left(J_{k}\right)=\mu\left(C_{k}\right)$. We set $B_{k}=\bigcap_{j \geq k} C_{j}$. Then $B_{k}$ is $\mu$-measurable and $J_{k} \subset B_{k}$ and $\mu^{*}\left(J_{k}\right)=\mu\left(B_{k}\right)$. Therefore

$$
\lim _{k \rightarrow+\infty} \mu^{*}\left(J_{k}\right)=\lim _{k \rightarrow+\infty} \mu\left(B_{k}\right)=\mu\left(\bigcup_{k=1}^{\infty} B_{k}\right) \geq \mu\left(\bigcup_{k=1}^{\infty} J_{k}\right)=\mu(J) .
$$

On the other hand, since $\mu^{*}\left(J_{k}\right) \leq \mu(J)$, we have

$$
\lim _{k \rightarrow+\infty} \mu^{*}\left(J_{k}\right) \leq \mu(J) .
$$

Thus we obtain

$$
\lim _{k \rightarrow+\infty} \mu^{*}\left(J_{k}\right)=\mu(J)
$$

\section{Weighted weak $(1,1)$ estimates of Hardy-Littlewood maximal operators on homogeneous spaces}

We will prove in this section the weighted weak- $(1,1)$ inequality of the centered HardyLittlewood maximal operator on a metric space possesing a doubling Radon measure. We must emphasize that this type of inequality is well known. It is proved by Calderón ([2]). In this paper, we will prove the weighted weak- $(1,1)$ inequality with better constant than previously known (as far as we know). When $w \equiv 1$, we have the ordinary unweighted weak- $(1,1)$ inequality of the centered Hardy-Littlewood maximal operator, and even in this case, the proof of the main theorem gives a new proof the weak- $(1,1)$ boundness of the centered Hardy-Littlewood maximal operator. The author got some hints of this 
proof from Carlsson's paper ([3]) and Termini and Vitanza's paper ([12]). The reader should also notice that the method of the proof resembles to Theorem 2.4.

Theorem 3.1. Let $X$ be a metric space possseing a doubling Radon measure $\mu$. Let $w$ be an $A_{1}$-weight. Namely, there exists a positive number $c>0$ such that

$$
\frac{1}{\mu(B(x, r))} \int_{B(x, r)} w(y) d \mu(y) \leq c \cdot \operatorname{essinf}_{y \in B(x, r)} w(y)
$$

holds for any ball $B(x, r)$. Let $d$ be an $A_{1}$-constant of $w$, and set

$$
e_{\lambda}=\inf \{e \mid w(B(x, \lambda r)) \leq e \cdot w(B(x, r)), \forall x \in X, \forall r>0\}
$$

Set $e=\lim _{\lambda \rightarrow 2+} e_{\lambda}$. Then

$$
w(\{x \mid \operatorname{Mf}(x)>\lambda\}) \leq \frac{d \cdot e}{\lambda} \int_{X}|f(x)| w(x) d \mu(x)
$$

holds.

Proof. Let $R>0$ be fixed. We will show that

$$
w\left(\left\{x \mid M_{R} f(x)>\lambda\right\}\right) \leq \frac{d \cdot e}{\lambda} \int_{X}|f(x)| w(x) d \mu(x)
$$

holds for any $f \in L^{1}(X, \mu)$. Let $o \in X$ be a fixed point. Let $r>0$ be a positive number. Set

$$
J=\{x \mid d(o, x)<r\} \cap\left\{x \mid M_{R} f(x)>\lambda\right\} .
$$

We choose $r_{x} \leq R$ for each $x \in J$ such that

$$
\frac{1}{\mu\left(B\left(x, r_{x}\right)\right)} \int_{B\left(x, r_{x}\right)}|f| d \mu>\lambda
$$

holds. Set $J_{n}=\left\{x \in K \mid r_{x}>1 / n\right\}$. Let $0<\theta<1$. Set $R_{1}=\sup _{x \in K_{n}} r_{x}$. Take $x_{1} \in J_{n}$ such that

$$
\frac{1}{\mu\left(B\left(x_{1}, r_{x_{1}}\right)\right)} \int_{B\left(x_{1}, r_{x_{1}}\right)}|f| d \mu>\lambda
$$

holds. Then,

$$
\begin{aligned}
& \frac{d \cdot e_{2 / \theta}}{\lambda} \int_{B\left(x_{1}, r_{x_{1}}\right)}|f| w d \mu \\
& \quad \geq \frac{d \cdot e_{2 / \theta}}{\lambda} \operatorname{essinf}_{B\left(x_{1}, r_{x_{1}}\right)} w \int_{B\left(x_{1}, r_{x_{1}}\right)}|f| d \mu \geq \frac{d \cdot e_{2 / \theta}}{\lambda} \operatorname{essinf}_{B\left(x_{1}, r_{x_{1}}\right)} w \cdot \lambda \mu(B(x, r)) \\
& \quad \geq e_{2 / \theta} \int_{B\left(x_{1}, r_{x_{1}}\right)} w d \mu \geq \int_{B\left(x,(2 / \theta) r_{x_{1}}\right)} w d \mu .
\end{aligned}
$$



Then,

Set $R_{2}=\sup _{x \in J_{n} \backslash B\left(x_{1},(2 / \theta) r_{x_{1}}\right)} r_{x}$. There exists $x_{2} \in J_{n} \backslash B\left(x_{1},(2 / \theta) r_{x_{1}}\right)$ such that $\theta R_{2}<r_{x_{2}}$.

$$
\frac{d \cdot e_{2 / \theta}}{\lambda} \int_{B\left(x_{2}, r_{x_{2}}\right)}|f| w d \mu \geq \int_{B\left(x,(2 / \theta) r_{x_{2}}\right)} w d \mu
$$

We will take $x_{i}$ in the same way. Then,

$$
\frac{d \cdot e_{2 / \theta}}{\lambda} \int_{B\left(x_{i}, r_{x_{i}}\right)}|f| w d \mu \geq \int_{B\left(x,(2 / \theta) r_{x_{i}}\right)} w d \mu .
$$

Then, we finally have

$$
B\left(x_{1}, \frac{2}{\theta} r_{x_{1}}\right) \cup B\left(x_{2}, \frac{2}{\theta} r_{x_{2}}\right) \cup \cdots \cup B\left(x_{n}, \frac{2}{\theta} r_{x_{n}}\right) \supset J_{n} .
$$

Adding the previous inequalities, we have

$$
\frac{d \cdot e_{2 / \theta}}{\lambda} \int_{X}|f| w d \mu \geq w^{*}\left(J_{n}\right) .
$$

Here, $w^{*}$ is the outer measure associated with the weighted measure $w$. Letting $n \rightarrow+\infty$, we have

$$
w(J) \leq \frac{d \cdot e_{2 / \theta}}{\lambda} \int_{X}|f| w d \mu .
$$

Since we can choose $r>0$ arbitrary in the definition of $J$, we have

$$
w(\{x \mid \operatorname{Mf}(x)>\lambda\}) \leq \frac{d \cdot e_{2 / \theta}}{\lambda} \int_{X}|f| w d \mu .
$$

Letting $\theta \rightarrow 1+$, we have

$$
w(\{x \mid \operatorname{Mf}(x)>\lambda\}) \leq \frac{d \cdot e}{\lambda} \int_{X}|f| w d \mu .
$$

Remark 3.2. Carlsson's result ([3]), combined with the result of Trinidad Menarguez and Soria ([13]), implies that the weak- $(1,1)$ constant of the centered Hardy-Littlewood maximal operator with respect to Eucledian balls on $\mathbf{R}^{\mathbf{n}}$ with Lebeague measure is less than or equal to $2^{n}$. The above theorem can be regarded as a generalization of this fact.

\section{Weak $(1,1)$ estimates of the one-sided Hardy-Littlewood maximal operators on the real line with respect to an absolutely continuous measure}

In [1], Bernal proved that one-sided Hardy-Littlewood maximal operator on the real line associated with any Borel measure is weak- $(1,1)$ bounded with constant 1 . We will prove here by a method different from A. Bernal's that one-sided Hardy-Littlewood maximal operator on the real line associated with absolutely continuous measure is weak- $(1,1)$ bounded with constant 1 . After I had found this proof of the result by myself, I knew 
that this kind of proof is in fact already known. See Sierpinski ([11]), Wiener ([14]) and Muckenhoupt-Stein ([8]). Especially, B. Muckenhoupt-E. M. Stein vaguely pointed out this kind of proof. However, since this method of the proof of the result may be regarded as the easiest example of our method, we will include the proof of it here for reference. We will define the one-sided Hardy-Littlewood maximal operator $M_{\mu,+}$ with respect to the absolutely continuous measure $\mu$ on $\mathbf{R}$ such that any interval which has nonzero length has nonzero $\mu$-measure.

Definition 4.1. Let $\mu$ be an absolutely continuous measure on $\mathbf{R}$ such that any interval which has nonzero length has nonzero $\mu$-measure. We define a one-sided maximal function $M_{\mu,+} f(x)$ for a locally integrable function $f$ on $\mathbf{R}$ with respect to the measure $\mu$ as follows:

$$
M_{\mu,+} f(x)=\sup _{h>0} \frac{1}{\mu([x, x+h))} \int_{x}^{x+h}|f| d \mu .
$$

TheORem 4.2. Let $\mu$ be an absolutely continuous measure on $\mathbf{R}$ such that any interval which has nonzero length has nonzero $\mu$-measure. Let $M_{\mu,+} f(x)$ be a one-sided maximal function of an integrable function $f$. Then

$$
\mu\left(\left\{x \mid M_{\mu,+} f(x)>\lambda\right\}\right) \leq \frac{1}{\lambda}\|f\|_{\mu, 1}
$$

holds for any $f \in L^{1}(\mu)$.

Proof. Since $\mu$ is absolutely continuous, $\mu$ is a Radon measure on $\mathbf{R}$. Thus the set $\{x \mid$ $\left.M_{+} f(x)>\lambda\right\}$ is an open set. Let $K$ be an arbitrary compact set contained in the set $\{x \mid$ $\left.M_{+} f(x)>\lambda\right\}$. We can choose for each $x \in K, h_{x}>0$ such that the inequality $1 /\left(\mu\left(\left[x, x+h_{x}\right)\right)\right) \int_{x}^{x+h_{x}}|f| d \mu>\lambda$ holds. Set $K_{n}=\left\{x \in K \mid h_{x}>1 / n\right\}$. Set $\inf K_{n}=a$, $\sup K_{n}=b$. Set

$$
m=[n|b-a|+1]
$$

Here, [ [ ] is a Gauss symbol. Let $\epsilon>0$ be an arbitrary positive number. Then, by the absolute continuity of the measure $\mu$, there exists a positive number $\delta>0$ such that if $|E|<\delta$, then $\mu(E)<\epsilon$. (Here, $|E|$ denotes the Lebesgue measure of the set $E$.) There exists a point $x_{1} \in K_{n}$ such that $x_{1}<\inf K_{n}+\delta /(m+1)$. By the definition of $K_{n}$, the inequality

$$
\frac{1}{\mu\left(\left[x_{1}, x_{1}+h_{x_{1}}\right)\right)} \int_{x_{1}}^{x_{1}+h_{x_{1}}}|f| d \mu>\lambda,
$$

holds. If $K_{n} \subset\left(-\infty, x_{1}+h_{x_{1}}\right)$, we stop here. If not, there exists a point $x_{2}$ such that $x_{2}<$ $\inf \left(K_{n} \backslash\left(-\infty, x_{1}+h_{x_{1}}\right)\right)+\delta /(m+1)$, and the inequality

$$
\frac{1}{\mu\left(\left[x_{2}, x_{2}+h_{x_{2}}\right)\right)} \int_{x_{2}}^{x_{2}+h_{x_{2}}}|f| d \mu>\lambda,
$$


holds. If $K_{n} \subset\left(-\infty, x_{2}+h_{x_{2}}\right)$, we stop here. If not, there exists a point $x_{3}$ such that $x_{3}<$ $\inf \left(K_{n} \backslash\left(-\infty, x_{2}+h_{x_{2}}\right)\right)+\delta /(m+1)$, and the inequality

$$
\frac{1}{\mu\left(\left[x_{3}, x_{3}+h_{x_{3}}\right)\right)} \int_{x_{3}}^{x_{3}+h_{x_{3}}}|f| d \mu>\lambda,
$$

holds. We will proceed in the same way. Then finally, for $x_{k} \in K_{n}$ we have $K_{n} \subset\left(-\infty, x_{k}+\right.$ $h_{x_{k}}$ ), and we have the inequality

$$
\frac{1}{\mu\left(\left[x_{k}, x_{k}+h_{x_{k}}\right)\right)} \int_{x_{k}}^{x_{k}+h_{x_{k}}}|f| d \mu>\lambda .
$$

Adding the inequalities about the integral, we have

$$
\mu^{*}\left(K_{n}\right)-\epsilon<\frac{1}{\lambda} \int_{\mathbf{R}}|f| d \mu
$$

Here, $\mu^{*}$ is the outer measure associated with measure $\mu$. Thus

$$
\mu^{*}\left(K_{n}\right) \leq \frac{1}{\lambda} \int_{\mathbf{R}}|f| d \mu
$$

Letting $n \rightarrow+\infty$, we have

$$
\mu(K) \leq \frac{1}{\lambda} \int_{\mathbf{R}}|f| d \mu
$$

Since $K$ is an arbitrary compact set contained in the set $\left\{x \mid M_{\mu,+} f(x)>\lambda\right\}$ and since $\mu$ is a Radon measure, we have

$$
\mu\left(\left\{x \mid M_{\mu,+} f(x)>\lambda\right\}\right) \leq \frac{1}{\lambda}\|f\|_{\mu, 1} .
$$

\section{Acknowledgments}

This work was done while the author was at the University of Tokyo. He would like to express deep gratitude to Professor Hitoshi Arai for his warm encouragements and patience. He also thanks Dr. Xu Bin and Mr. Yoshihiro Sawano for helpful dicussions with him.

\section{References}

[1] A. Bernal, A note on the one-dimensional maximal function, Proceedings of the Royal Society of Edinburgh. Section A. Mathematics 111 (1989), no. 3-4, 325-328.

[2] A.-P. Calderón, Inequalities for the maximal function relative to a metric, Studia Mathematica 57 (1976), no. 3, 297-306.

[3] H. Carlsson, A new proof of the Hardy-Littlewood maximal theorem, The Bulletin of the London Mathematical Society 16 (1984), no. 6, 595-596. 
[4] R. R. Coifman and G. Weiss, Analyse harmonique non-commutative sur certains espaces homogènes, Lecture Notes in Mathematics, vol. 242, Springer, Berlin, 1971.

[5] L. C. Evans and R. F. Gariepy, Measure Theory and Fine Properties of Functions, Studies in Advanced Mathematics, CRC Press, Florida, 1992.

[6] G. H. Hardy and J. E. Littlewood, A maximal theorem with function-theoretic applications, Acta Mathematica 54 (1930), 81-116.

[7] A. D. Melas, The best constant for the centered Hardy-Littlewood maximal inequality, Annals of Mathematics. Second Series 157 (2003), no. 2, 647-688.

[8] B. Muckenhoupt and E. M. Stein, Classical expansions and their relation to conjugate harmonic functions, Transactions of the American Mathematical Society 118 (1965), 17-92.

[9] F. Nazarov, S. Treil, and A. Volberg, Weak type estimates and Cotlar inequalities for CalderónZygmund operators on nonhomogeneous spaces, International Mathematics Research Notices 1998 (1998), no. 9, 463-487.

[10] Y. Sawano, Sharp estimates of the modified Hardy-Littlewood maximal operator on the nonhomogeneous space via covering lemmas, Hokkaido Mathematical Journal 34 (2005), 435-458.

[11] W. Sierpinski, Un lemme métrique, Fundamenta Mathematicae 4 (1923), 201-203 (French).

[12] D. Termini and C. Vitanza, Weighted estimates for the Hardy-Littlewood maximal operator and Dirac deltas, The Bulletin of the London Mathematical Society 22 (1990), no. 4, 367-374.

[13] M. Trinidad Menarguez and F. Soria, Weak type $(1,1)$ inequalities of maximal convolution operators, Rendiconti del Circolo Matematico di Palermo. Serie II 41 (1992), no. 3, 342-352.

[14] N. Wiener, The ergodic theorem, Duke Mathematical Journal 5 (1939), 1-18.

Yutaka Terasawa: Department of Mathematics, Hokkaido University, Sapporo 060-0810, Japan E-mail address: yutaka@math.sci.hokudai.ac.jp 